\title{
Involuntary Movements Induced and Remitted by Indirect Bypass Surgery in a Patient with Moyamoya Disease
}

\author{
Takao Yanagawa, Tetsuyoshi Horiuchi, Yosuke Hara, Yu Fujii, Kazuhiro Hongo \\ Department of Neurosurgery, Shinshu University School of Medicine, Matsumoto, Japan \\ Email: takao@shinshu-u.ac.jp
}

Received July 23, 2012; revised August 29, 2012; accepted September 13, 2012

\begin{abstract}
Involuntary movement is an uncommon manifestation of moyamoya disease. We describe a 12-year-old boy who suffered from involuntary movement after the first surgery but it improved dramatically after the second surgery. Initially, the patient underwent an encephalo-duro-arterio-myosynangiosis on the right side and encephalo-duro-periostealsynangiosis on the bifrontal side, which ameliorated his motor weakness in his left upper extremity. However, involuntary movement appeared in his right upper extremity. Secondly, the patient underwent encephalo-duro-arterio-myo-periostealsynangiosis on the left side. Thereafter, the patient's involuntary movement completely resolved. The involuntary movement might occur due to the unequal distribution of the blood flow in the basal ganglia.
\end{abstract}

Keywords: Involuntary Movement; Indirect Bypass Surgery; Moyamoya Disease

\section{Introduction}

Moyamoya disease, meaning a "hazy puff of smoke" in Japanese, is a chronic disease with progressive occlusion of the circle of Willis arteries that leads to the development of collateral vessels, which usually presents as recurrent episodes of transient ischemic attacks or strokes. The patients with moyamoya disease usually present with a variety of clinical symptoms due to ischemia or intracranial hemorrhage. Involuntary movement is a rare symptom associated with moyamoya disease [1]. We report here a unique case of involuntary movement appeared after the first indirect bypass surgery; however, it disappeared after the second indirect bypass surgery in a 12-year-old boy with moyamoya disease.

\section{Case Report}

The patient described in this report is a 12-year-old boy who experienced motor weakness in his left upper extremity provoked by conditions of hyperventilation 3 years before we examined him. Furthermore, one year later, similar weakness also observed in his right upper extremity and they had become more frequent. He was taken to a local hospital where neuroimages showed bilateral internal carotid artery stenosis. He was then referred to us.

On presentation, no neurological deficits were observed. Magnetic resonance (MR) imagings showed development of fine vascular plexus in the base of the brain, but no infarct or hemorrhage (Figure 1). Cerebral an- giography demonstrated severe stenosis of the distal internal carotid arteries with numerous collateral vessels in the vicinity of the stenosis. These features were consistent with moyamoya disease (Figure 2). Although brain $\left[{ }^{99 \mathrm{~m}} \mathrm{Tc}\right]$-hexamethylpropyleneamine oxime single-photon emission computed tomography (SPECT) revealed no obvious perfusion defects, surgical treatment was scheduled for frequent transient ischemic attacks.

Initially, the patient underwent encephalo-duro-arteriomyosynangiosis on the right middle cerebral artery (MCA) territory and encephalo-duro-periostealsynangiosis on the bifrontal anterior cerebral artery (ACA) territory, which ameliorated his transient motor weakness in the left side [2]. However, involuntary movement appeared in his right upper extremity without symptoms in his lower extremities three months after the surgery. The type of movement is irregular, quick and choreic. Brain MR imagings revealed no newly developed infarcted and hemorrhagic lesions. Secondly, he underwent encephaloduro-arterio-myo-periostealsynangiosis on the left MCA territory. Involuntary movement disappeared within one month after the second surgery. The patient has been free of transient motor weakness and involuntary movements for 22 months since the second surgery. Postoperative angiography was not performed because he refused it.

\section{Discussion}

Involuntary movement is a rare clinical feature of moyamoya disease. The pathophysiology of involuntary movement is not yet fully understood. In most cases of 

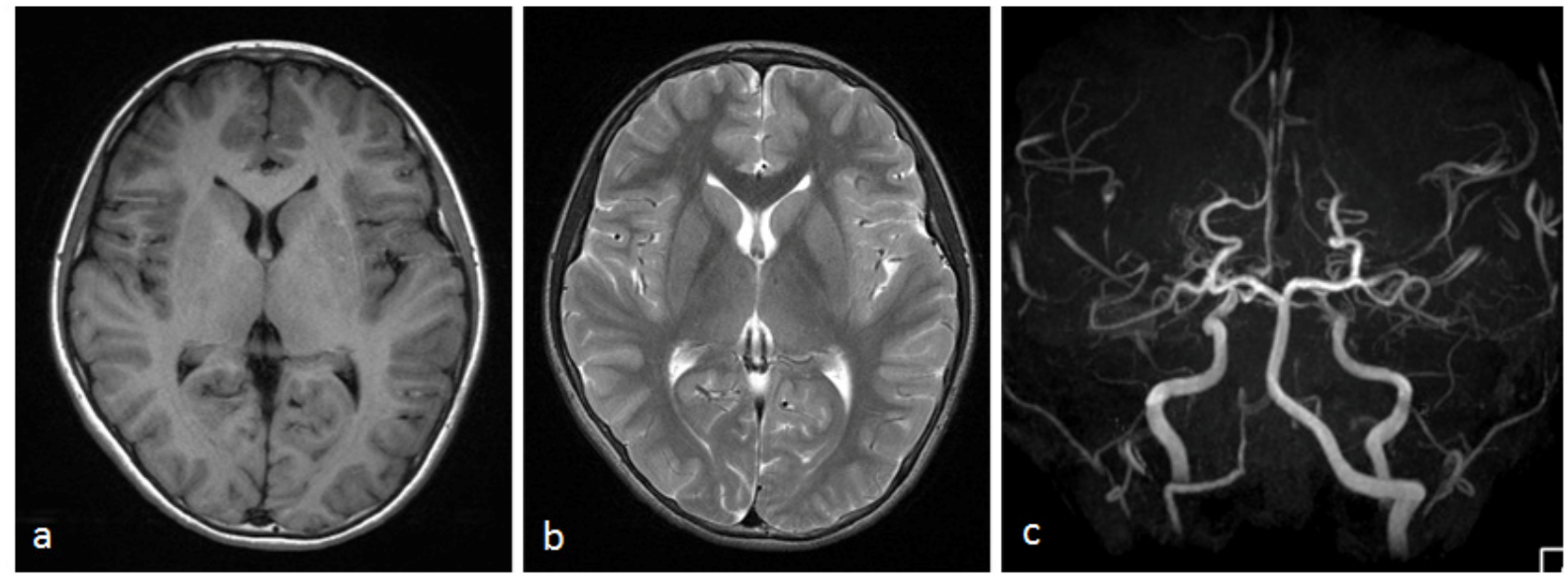

Figure 1. Preoperative axial T1-weighted (a), T2-weighted (b) magnetic resonance imagings, and magnetic resonance angiogram (c), showing the development of fine vascular plexus in the base of the brain, but no infarction or hemorrhage associated with moyamoya disease.
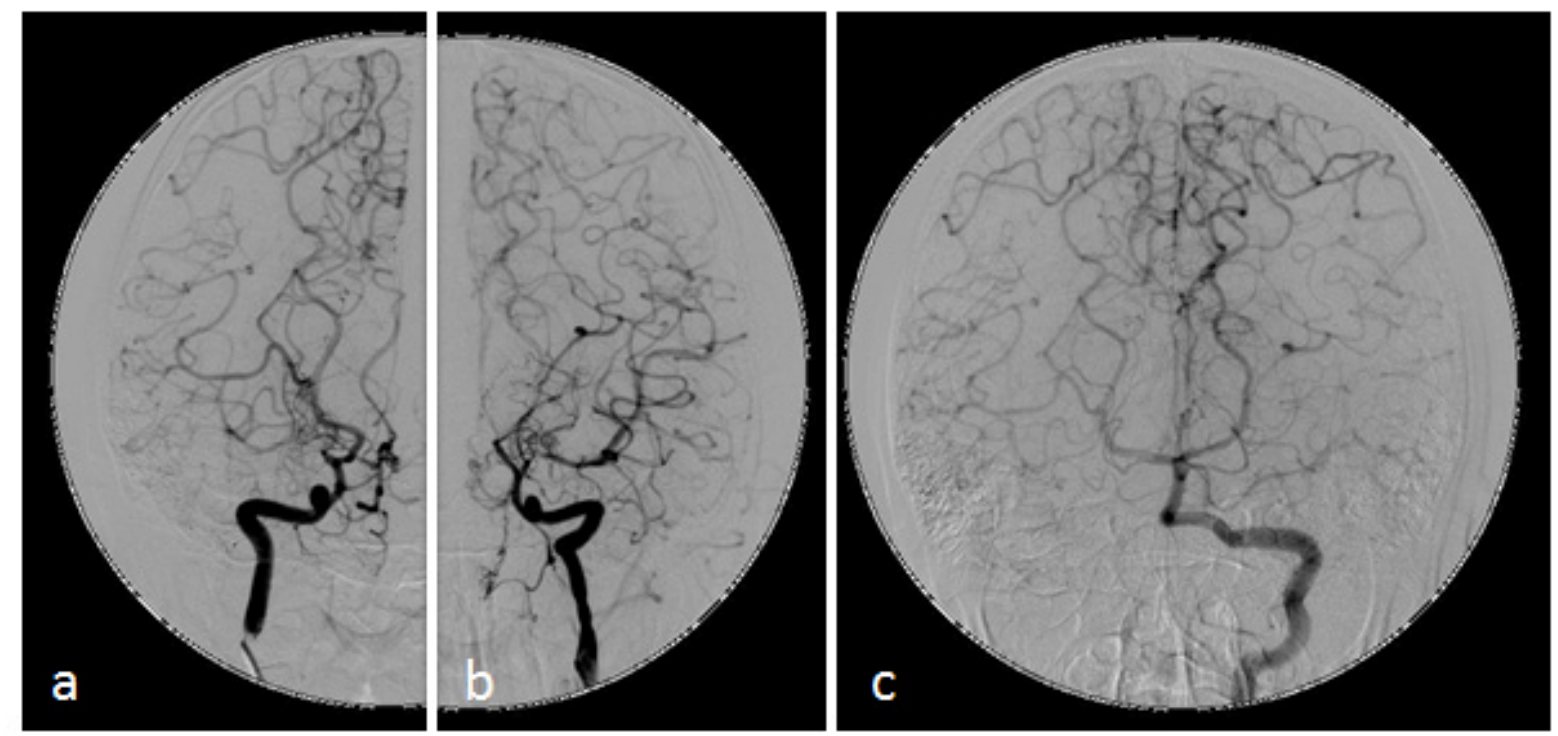

Figure 2. Preoperative anterior-posterior views of right (a) and left (b) carotid angiograms demonstrating severe stenosis that starts at the distal internal carotid arteries and the proximal anterior and middle cerebral arteries with numerous collateral vessels in the vicinity of the stenosis. Preoperative anterior-posterior (c) angiogram of the left vertebral artery showing an extensive network of collaterals which involves leptomeningeal vessels and collaterals from the posterior cereberal artery.

involuntary movement related with moyamoya disease, however, there are contralateral hemispheric lesions in areas such as the caudate nucleus, putamen, globus pallidus, subthalamic nucleus, parts of thalamus, and subcortical white matter which form cortico-basal ganglia loop, although there are several exceptions [3-5].

Several cases of involuntary movement in a patient with moyamoya disease have recently been reported. Table 1 summarizes clinical characteristics of 13 patients with moyamoya disease with involuntary movement including the present case [6-16]. There were 2 men (15.4\%) and 11 women (84.6\%) identified with a mean age of 21.3 years ranging from 7 to 54 . The responsible lesions vary on individuals. Surgical vascular reconstruction and medication therapy are effective for involuntary movement such as chorea in all patients. By contrast, in the present case, involuntary movement which appeared after the first indirect bypass surgery disappeared completely by the second indirect bypass surgery. The present case related with surgical therapies has not been reported previously. Involuntary movement has been previously described in patients with moyamoya disease and mostly appears relating to a focal reduction in perfusion reserve. However, in this case, there are no obvious ischemic and hemorrhagic lesions on SPECT studies preoperatively as well as postoperatively [1]. Therefore, 
Table 1. Cases of chorea in patients with moyamoya disease.

\begin{tabular}{ccccc}
\hline Author [ref. number] & Age (yrs) & Infarct on MRI & CBF studies & Effective therapy \\
\hline Tsuchiyama et al. [14] & 33/Female & CN & CN, P & Medical therapy \\
Hosoya et al. [5] & 9/Female & Fr, P, O & BG & STA-MCA anastomosis \\
Unno et al. [15] & 16/Female & Fr & Fr, P, BG & Chorea during pregnancy, \\
Han et al. [3] & 29/Female & Fr & Fr, T, BG & EDAS \\
Lyoo et al. [9] & 22/Female & Fr, T, P & Fr, T, P & Spontaneous resolution \\
Miura et al. [10] & 54/Male & No infarcts & T, P, S & Haloperidol \\
Hong et al. [4] & 20/Female & CS & Fr, BG & STA-MCA anastomosis \\
Im et al. [6] & 7/Female & Fr & Hemisphere & EDAS \\
Im et al. [6] & 13/Female & Fr, P & PBZ & EDS, EGS \\
Kim et al. [8] & 8/Female & No infarcts & Fr, P & EDAMS \\
Zheng et al. [16] & 15/Female & Fr, P, O & Fr, T, P, O & Fr, T, P, S \\
Kamijo et al. [7] & 31/Female & No infarcts & No defects & STA-MCA anastomosis, EDAMS \\
Present case & 13/Male & No infarcts & First: EDAMS, EPS & Second: EDAMPS \\
\hline
\end{tabular}

F: woman, M: man, CN: caudate nucleus, Fr: frontal lobe, T: temporal lobe, P: parietal lobe, BG: basal ganglia, S: striatum, PBZ: posterior border zone, MRI: magnetic resonance imaging, EDAS: encephalo-duro-arteriosynangiosis, EDAMS: encephalo-duro-arterio-myosynangiosis, EGS: encephalo-galeosynangiosis, EPS: encephalo-periostealsynangiosis, EDAMS: encephalo-duro-arterio-myo-periostealsynangiosis, STA: superficial temporal artery, MCA: middle cerebral artery, ref.: reference.

it is difficult to clarify the mechanism of the present involuntary movement associated with focal blood flow impairment. We speculate that some interruption of the cortico-basal ganglia loop due to unequal distribution of the blood flow might cause involuntary movement in his right upper extremity, because inadequate blood flow to the left MCA territory appeared after the patient underwent indirect revascularization on the right MCA territory and on the bifrontal ACA [6].

In this report, we described the case of involuntary movements following the first indirect bypass surgery and remitted by the second direct bypass surgery in moyamoya disease.

\section{REFERENCES}

[1] S.-H. Im, C. W. Oh, O.-K. Kwon, B.-K. Cho, Y.-S. Chung and D. H. Han, "Involuntary Movement Induced by Cerebral Ischemia: Pathogenesis and Surgical Outcome,” Journal of Neurosurgery, Vol. 100, No. 5, 2004, pp. 877-882. doi:10.3171/jns.2004.100.5.0877

[2] J. H. Park, S. Y. Yang, Y. N. Chung, J. E. Kim, S. K. Kim, D. H. Han and B. K. Cho, "Modified Encephaloduroarteriosynangiosis with Bifrontal Encephalogaleoperiosteal Synangiosis for the Treatment of Pediatric Moyamoya Disease. Technical Note," Journal of Neurosur- gery, Vol. 106, No. 3, 2007, pp. 237-242.

[3] G. E. Alexander and M. D. Crutcher, "Functional Architecture of Basal Ganglia Circuits: Neural Substrates of Parallel Processing," Trends in Neurosciences, Vol. 13, No. 7, 1990, pp. 266-271. doi:10.1016/0166-2236(90)90107-L

[4] G. E. Alexander, M. R. De Long and P. L. Strick, "Parallel Organization of Functionally Segregated Circuits Linking Basal Ganglia and Cortex," Annual Review of Neuroscience, Vol. 9, 1986, pp. 357-381. doi:10.1146/annurev.neuro.9.1.357

[5] S. H. Han, Y. G. Kim, S. H. Cha and S. Y. Chung, "Moyamoya Disease Presenting with Singing Induced Chorea,” Journal of Neurology, Neurosurg \& Psychiatry, Vol. 69, No. 6, 2000, pp. 833-834. doi:10.1136/jnnp.69.6.833

[6] Y. H. Hong, T. B. Ahn, C. W. Oh and B. S. Jeon, "Hemichorea as an Initial Manifestation of Moyamoya Disease: Reversible Striatal Hypoperfusion Demonstrated on Single Photon Emission Computed Tomography," Movement Disorders, Vol. 17, No. 6, 2002, pp. 13801383. doi: $10.1002 / \mathrm{mds} .10245$

[7] K. Nakano, T. Kayahara, T. Tsutsumi and H. Ushiro, "Neural Circuits and Functional Organization of the Striatum,” Journal of Neurology, Vol. 247, 2000, pp. V1V15. doi:10.1007/PL00007778

[8] M. Hosoya, "Moyamoya Disease Presenting Initially with Mental Disturbance," No to Hattatsu, Vol. 29, No. 6, 
1997, pp. 471-475.

[9] K. Kamijo and T. Matsui, "Dramatic Disappearance of Moyamoya Disease-Induced Chorea after Indirect Bypass Surgery,” Neurologia Medico-Chirurgica, Vol. 48, No. 9, 2008, pp. 390-393. doi:10.2176/nmc.48.390

[10] Y. O. Kim, T. S. Kim, Y. J. Woo, C. J. Kim and C. K. Oh, "Moyamoya Disease-Induced Hemichorea Corrected by Indirect Bypass Surgery," Pediatrics International, Vol. 48, No. 5, 2006, pp. 504-506. doi:10.1111/j.1442-200X.2006.02248.X

[11] C. H. Lyoo, S. H. Oh, J. Y. Joo, T. S. Chung and M. S. Lee, "Hemidystonia and Hemichoreoathetosis as an Initial Manifestation of Moyamoya Disease," Archives of Neurology, Vol. 57, No. 10, 2000, pp. 1510-1512. doi:10.1001/archneur.57.10.1510

[12] T. Miura, M. Kobayashi, M. Sonoo, K. Isii and T. Shimizu, "An Adult Case of Moyamoya Disease Presenting with Transient Hemichorea,” Rinsho Shinkeigaku, Vol. 42, No. 1, 2002, pp. 45-47.
[13] K. Spengos, G. Tsivgoulis, P. Toulas, K. Vemmos, D. Vassilopoulos and M. Spengos, "Hyperventilation-Enhanced Chorea as a Transient Ischaemic Phenomenon in a Patient with Moyamoya Disease,” European Neurology, Vol. 51, No. 3, 2004, pp. 172-175. doi:10.1159/000077665

[14] M. Tsuchiyama, K. Kawabata, O. Uyama, H. Tachibana and H. Sugita, "Hemichorea induced by Cerebrovascular Disease,” Nippon Iji Shinpo, Vol. 3534, 1992, pp. 43-45.

[15] S. Unno, M. Iijima, M. Osawa, S. Uchiyama and M. Iwata, "A Case of Chorea Gravidarum with Moyamoya Disease,” Rinsho Shinkeigaku, Vol. 40, No. 4, 2000, pp. 378-382.

[16] W. Zheng, M. Wanibuchi, T. Onda, H. Liu, I. Koyanagi, K. Fujimori and K. Houkin, "A Case of Moyamoya Disease Presenting with Chorea," Child's Nervous System, Vol. 22, No. 3, 2006, pp. 274-278. doi:10.1007/s00381-004-1104-2 